\title{
Effectiveness of Low Cut Modified Amplification using Receiver in the Canal Hearing Aid in Individuals with Auditory Neuropathy Spectrum Disorder
}

\author{
Prashanth Prabhu ${ }^{1}$ Animesh Barman ${ }^{1}$ \\ ${ }^{1}$ Department of Audiology, All India Institute of Speech and Hearing \\ Ringgold Standard Institution, Mysore, Karnataka, India \\ Int Arch Otorhinolaryngol 2017;21:243-249.
}

\author{
Address for correspondence Prashanth Prabhu, MSc, Department of \\ Audiology, All India Institute of Speech and Hearing, Naimisham \\ Campus Manasagangothri, Mysore, Karnataka 570006, India \\ (e-mail: prashanth.audio@gmail.com).
}

\begin{abstract}
Introduction The studies on hearing aid benefit in individuals with auditory neuropathy spectrum disorder (ANSD) shows limited benefit. Low cut modified amplification is found to be effective in few individuals with ANSD. With advancement in technology, receiver in the canal (RIC) hearing aids have proven to be more effective than traditional behind the ear (BTE) hearing aids.

Objective Thus, the present study attempts to determine the effectiveness of low cut modified amplification using RIC and BTE.

Method Twenty participants with ANSD were fitted with BTE and RIC using traditional and low cut modified amplification. We divided them into good and poor performers based on unaided speech identification scores (SIS). We then compared aided SIS and aided benefit across conditions in good and poor performers with ANSD across both conditions using BTE and RIC.

Results The results of the study showed that the aided performance improved with low cut modified amplification in both BTE and RIC hearing aids. The improvement noticed with low-cut modified fitting with RIC was significant in more than BTE, especially in good performers with ANSD.

Keywords

- hearing aids

Conclusion The improved clarity and naturalness of sound with RIC may have led to

- hearing loss better aided scores and better acceptance of the hearing aid. Thus, low-cut modified amplification, preferably with RIC, needs to be attempted in fitting individuals with

- speech perception ANSD, especially in those with good unaided SIS in quiet.
\end{abstract}

\section{Introduction}

Auditory neuropathy spectrum disorder (ANSD) is as a retrocochlear disorder where otoacoustic emissions (OAE) are normal and auditory brainstem responses (ABR) are abnormal/absent. ${ }^{1,2}$ The prevalence rate of ANSD in Western countries reportedly varies from $11 \%$ to $0.5 \%{ }^{3-6}$ Among the Indian population, Kumar and Jayaram ${ }^{7}$ reported a prevalence of ANSD of 1 in 183 individuals diagnosed as having sensorineural hearing loss. Bhat, Kumar, and Sinha ${ }^{8}$ reported that the prevalence of ANSD in school-going children with hearing impairment is $\sim 2.47 \%$.

received

May 18, 2016

accepted

August 23, 2016

published online

November 22, 2016 10.1055/s-0036-1593471. ISSN 1809-9777.
The audiological profile of individuals with auditory neuropathy spectrum disorder is variable. The hearing loss in individuals with ANSD can range from normal hearing to profound degree and predominantly having bilateral symmetrical hearing loss. ${ }^{6,9}$ The majority of individuals with ANSD show reverse sloping or peaked audiogram configuration. ${ }^{2,7,10}$ The speech recognition deficits in individuals with ANSD are out of proportion to their pure-tone thresholds. ${ }^{711}$ The rehabilitation strategies used in individuals with ANSD include the use of conventional hearing aid amplification, use of cochlear implants, and speech
Copyright $\odot 2017$ by Thieme Revinter Publicações Ltda, Rio de Janeiro, Brazil
License terms

()(1) $\Theta \circledast$ 
enhancement techniques. Majority of the studies suggest that hearing aids are of limited usefulness in individuals with ANSD. $^{12}$

The limited benefit from hearing aids in individuals with ANSD could be because of inappropriate fitting of the device. Zeng et $\mathrm{al}^{13}$ suggested that individuals with ANSD require a unique speech processing in hearing aids which should compensate for affected spectral and temporal processing in addition to amplifying and making the sounds audible. Prabhu, Avilala, and Barman ${ }^{14}$ reported very poor speech identification scores with low-pass filtered speech, but less deterioration in scores for high-pass filtered speech in individuals with ANSD. They suggested that individuals with ANSD use mainly high frequency information to perceive speech. However, in the conventional hearing aid amplification, low frequencies are amplified more since most of them have reverse sloping or peaked audiogram configuration. ${ }^{2,7,10}$ Thus, conventional amplification could unnecessarily amplify low frequencies which may not be used by individuals with ANSD. ${ }^{14,15}$ Based on the above rationale, Barman, Sinha, and Prabhu ${ }^{12}$ attempted to study the effectiveness of frequency specific amplification only at high frequencies in individuals with ANSD. They reported that frequency specific amplification was shown to be effective compared with traditional hearing aid amplification in individuals with ANSD. They considered only BTE hearing aid with 4 and 16 channels with custom ear mold. With the advancement in technology, hearing aid has gone through several modifications and needs to be explored in ANSD group to check for usefulness.

Receiver in the Canal (RIC) hearing aids reportedly improve the clarity of speech compared with traditional behind the ear (BTE) hearing aids. ${ }^{16,17}$ With the ear canal open, hearing aid users generally report a more natural and clear sound quality. ${ }^{18-20}$ The larger distance between the receiver and microphone leads to less feedback and gives an opportunity to use open dome fitting, which reduces the chance of occlusion in the hearing aid users. Thus, RIC hearing aid with open dome provides sufficient venting to remove the low frequencies. The inherent advantages of open fit RIC hearing aid with low-cut hearing aid modification may enhance sound clarity, which could benefit individuals with ANSD and needs to be further explored. Thus, the present study attempts to determine whether there is any difference in aided speech perception with open fit RIC hearing aid compared with BTE hearing aid with regular mold using lowcut modified amplification of hearing aid fitting in individuals with ANSD. In addition, there are studies reporting that speech perception also depends on unaided speech identification scores. $^{21-23}$ Narne and Vanaja ${ }^{22}$ suggested that individuals with ANSD can be divided as poor performers if the unaided speech identification scores (SIS) in quiet is less than $50 \%$ and good performers if the SIS is greater than $50 \%{ }^{21}$ Speech perception in noise scores ${ }^{21}$ and results of temporal modulation transfer function ${ }^{23}$ are reported to be relatively better in good performers compared with poor performers. Considering these perceptual differences, we also attempted to determine the benefit of low-cut modified amplification using BTE and RIC in good and poor performers with ANSD. Thus, the study attempted to determine the effect of conventional full band amplification and low-cut modified amplification on aided speech identification scores for BTE and open fit RIC hearing aid. In addition, we also attempted to determine the effect of low-cut modified amplification for good and poor performers of ANSD.

\section{Method}

\section{Participants}

We selected a total of 20 individuals (40 ears) with ANSD for the study, which included 10 males and 10 females, within the age ranges of 15 to 40 years (mean age of 25 years). All the participants had pure tone average thresholds (PTA, average of pure tone thresholds at $500 \mathrm{~Hz}, 1 \mathrm{kHz}, 2 \mathrm{kHz}$, and $4 \mathrm{kHz}$ ) ranging from mild (26-40 dB HL), moderate (41-55 dB), moderately severe (56-70 dB HL), and severe (71-90 dB HL) degree of hearing loss. ${ }^{24}$ They were diagnosed as having ANSD based on presence of transient evoked oto-acoustic emissions but absent auditory brainstem responses. A neurologist confirmed the diagnosis. All participants were native and fluent speakers of Kannada. They had no history and presence of middle ear pathology with A-type tympanogram ${ }^{25}$ and absent acoustic reflexes. The participants who obtained less than 50\% SIS at $40 \mathrm{~dB}$ SL in quiet were grouped as poor performers ( 24 ears) and above $50 \%$ were grouped as good performers (16 ears).

The demographic details and audiological findings of all the participants are given in -Table $\mathbf{1}$.

\section{Procedure}

We estimated pure tone air conduction (AC) and bone conduction $(\mathrm{BC})$ thresholds using modified Hughson and Westlake procedure. ${ }^{26}$ We obtained AC thresholds for pure tones from $250 \mathrm{~Hz}$ to $8 \mathrm{kHz}$ and $\mathrm{BC}$ thresholds from $250 \mathrm{~Hz}$ to $4 \mathrm{kHz}$ in octave frequencies. We used a two-channel diagnostic audiometer to obtain air conduction and bone conduction pure tone thresholds and speech identification scores. We obtained the unaided speech identification scores for phonemically balanced words in Kannada. Recorded word lists were routed from a personal computer through a two-channel diagnostic audiometer at $40 \mathrm{~dB}$ SL (re: SRT). We used a Tympstar (GSI-TS) emittance meter (Grason-Stadler Inc., Eden Prairie, MN, U.S.A.) was used for emittance testing. We tested each ear of the participant to obtain tympanogram and acoustic reflexes for a probe tone frequency of $226 \mathrm{~Hz}$. We measured acoustic reflexes using 500, 1000, 2000, and $4000 \mathrm{~Hz}$ pure tones presented to both ipsi-lateral and contra-lateral ears. We used Otodynamics ILO v.6 OAE analyzer (Otodynamics Ltd, Herts, UK) to obtain Transient Evoked Otoacoustic Emissions (TEOAEs). After ensuring adequate probe fit, we measured TEOAEs for non-linear click trains presented at $80 \mathrm{~dB}$ pe SPL. Waveform reproducibility of more than $50 \%{ }^{27}$ and an overall signal to noise ratio of more than $3 \mathrm{~dB} \mathrm{SPL}^{28}$ at least at two frequency bands was required to be considered as presence of TEOAEs.

Biologic Navigator Pro (Bio-logic, Mundelein, IL, U.S.A.) AEP system with ER 3A insert earphones was used to record 
Table 1 Demographic details and audiological findings of the 20 participants considered for the study

\begin{tabular}{|c|c|c|c|c|c|c|}
\hline Participants & Ear & Age & Gender & PTA & SIS & Configuration \\
\hline \multirow[t]{2}{*}{ P1 } & Right & \multirow[t]{2}{*}{18} & \multirow[t]{2}{*}{ Female } & 41.25 & $44 \%$ & Rising \\
\hline & Left & & & 70 & $32 \%$ & Rising \\
\hline \multirow[t]{2}{*}{$\mathrm{P} 2$} & Right & \multirow[t]{2}{*}{32} & \multirow[t]{2}{*}{ Male } & 57.5 & $72 \%$ & Flat \\
\hline & Left & & & 50 & $84 \%$ & Flat \\
\hline \multirow[t]{2}{*}{ P3 } & Right & \multirow[t]{2}{*}{24} & \multirow[t]{2}{*}{ Male } & 43.75 & $80 \%$ & Rising \\
\hline & Left & & & 50 & $84 \%$ & Rising \\
\hline \multirow[t]{2}{*}{$\mathrm{P} 4$} & Right & \multirow[t]{2}{*}{15} & \multirow[t]{2}{*}{ Female } & 38.75 & $68 \%$ & Flat \\
\hline & Left & & & 53.25 & $60 \%$ & Flat \\
\hline \multirow[t]{2}{*}{ P5 } & Right & \multirow[t]{2}{*}{32} & \multirow[t]{2}{*}{ Male } & 32.5 & $68 \%$ & Rising \\
\hline & Left & & & 40 & $72 \%$ & Rising \\
\hline \multirow[t]{2}{*}{ P6 } & Right & \multirow[t]{2}{*}{23} & \multirow[t]{2}{*}{ Male } & 47.5 & $12 \%$ & Flat \\
\hline & Left & & & 56.25 & $8 \%$ & Flat \\
\hline \multirow[t]{2}{*}{ P7 } & Right & \multirow[t]{2}{*}{19} & \multirow[t]{2}{*}{ Female } & 55 & $8 \%$ & Flat \\
\hline & Left & & & 30 & $20 \%$ & Flat \\
\hline \multirow[t]{2}{*}{ P8 } & Right & \multirow[t]{2}{*}{17} & \multirow[t]{2}{*}{ Male } & 50 & $16 \%$ & Flat \\
\hline & Left & & & 55 & $24 \%$ & Flat \\
\hline \multirow[t]{2}{*}{ P9 } & Right & 21 & Female & 25 & $68 \%$ & Rising \\
\hline & Left & & & 41.25 & $32 \%$ & Rising \\
\hline P10 & Right & 25 & Male & 43.75 & $0 \%$ & Flat \\
\hline & Left & & & 42.5 & $0 \%$ & Flat \\
\hline P11 & Right & 23 & Male & 68.75 & $60 \%$ & Rising \\
\hline & Left & & & 66.25 & $52 \%$ & Rising \\
\hline P12 & Right & 25 & Female & 50 & $12 \%$ & Flat \\
\hline & Left & & & 68.25 & $8 \%$ & Flat \\
\hline P13 & Right & 26 & Female & 50 & $8 \%$ & Rising \\
\hline & Left & & & 40 & $20 \%$ & Rising \\
\hline P14 & Right & 15 & Female & 28.75 & $16 \%$ & Flat \\
\hline & Left & & & 22.5 & $24 \%$ & \\
\hline P15 & Right & 35 & Female & 42.5 & $68 \%$ & Rising \\
\hline & Left & & & 52.5 & $32 \%$ & Rising \\
\hline P16 & Right & 33 & Female & 30 & $0 \%$ & Flat \\
\hline & Left & & & 23.75 & $0 \%$ & Rising \\
\hline P17 & Right & 25 & Male & 33.75 & $60 \%$ & Flat \\
\hline & Left & & & 30 & $52 \%$ & Flat \\
\hline P18 & Right & 40 & Female & 83.75 & $28 \%$ & Rising \\
\hline & Left & & & 82.5 & $32 \%$ & Rising \\
\hline P19 & Right & 35 & Male & 45 & $0 \%$ & Flat \\
\hline & Left & & & 43.75 & $0 \%$ & Flat \\
\hline P20 & Right & 18 & Male & 40 & $48 \%$ & Rising \\
\hline & Left & & & 63.75 & $80 \%$ & Rising \\
\hline
\end{tabular}


ABR. ABR was recorded with the clients seated on a reclining chair. We cleaned the skin surface on the two mastoids and forehead with skin abrasive. We used gold cup electrodes to record responses. We placed the electrodes with the help of skin conduction paste and used surgical plaster to hold the electrodes tightly on the respective places. We maintained absolute electrode impedance below $5 \mathrm{k} \Omega$ with inter electrode impedance below $2 \mathrm{k} \Omega$. Before starting the recording, participants were instructed to relax and refrain from extraneous body movements to minimize artifacts. We obtained single channel recordings with inverting electrode on the test ear mastoid (M2/M1), non-inverting electrode on the high forehead $(\mathrm{Fz})$, and ground electrode on the non-test ear mastoid (M1/M2). We recorded click-evoked ABR twice and replicated for $100 \mu \mathrm{sec}$ click stimuli delivered at a repetition rate of 11.1 clicks/second at $90 \mathrm{~dB} \mathrm{nHL}$. We obtained the recording for a total of 1500 sweeps and used a filter setting of $100 \mathrm{~Hz}$ to $3000 \mathrm{~Hz}$.

We used a 4-channel digital BTE and a 4-channel digital RIC hearing aid with open dome with similar features and from the same company to assess the effectiveness of full band and selective band amplification in individuals with ANSD. Both the hearing aids had four frequency bands with the facility to adjust the cut-off frequency for each band separately. The hearing aids had three programs in which three modes of amplification (conventional and low-cut modified amplification) could be stored. The compression parameters, noise reduction algorithms, feedback cancellation parameters, and directionality were kept constant. Both the hearing aids had the option to program for individuals with hearing impairment of various degrees. The specifications of the hearing aids are given in -Table $\mathbf{2}$.

We input the pure tone thresholds (from $250 \mathrm{~Hz}$ to $8 \mathrm{kHz}$ for air conduction and from $250 \mathrm{~Hz}$ to $4 \mathrm{kHz}$ for bone conduction) of the participants' test ear into the NOAH fitting software. We connected the hearing aid to the HI-PRO/NOAH link, which in turn was connected to a computer with the programming software. The hearing aid programming software detected the hearing aid and we selected the default prescriptive formula of NAL-NL1. We programmed the fourchannel BTE hearing aid and four-channel RIC hearing aid for conventional method and selective band amplification. The hearing aid was set to the first fit condition and gain provided at all the frequencies using conventional method was stored in the first program P1. The second program (P2) had low-cut modified amplification where the low frequency channel having center frequency of $250 \mathrm{~Hz}$ had $15 \mathrm{~dB}$ lesser gain compared with the P1. We determined the unaided and aided speech identification scores (SIS) for both BTE and RIC using conventional and low-cut modified amplification strategy. We used phonemically balanced bi-syllabic word lists with 25 lists of 25 words each developed by Manjula et al. ${ }^{29}$

We determined the aided speech identification scores at $40 \mathrm{~dB}$ HL for individuals having degree of hearing loss above mild and $30 \mathrm{~dB}$ HL for individuals having mild degree of hearing loss. The testing was performed in a double room setup with participant seated at a one-meter distance from the loudspeaker. The recorded word lists were presented through loudspeaker kept at $0^{\circ}$ azimuth in relation to the participant. We initially obtained unaided speech identification scores. Aided testing was done using two hearing aids in both conventional and low cutmodified amplification condition for both ears using BTE and RIC. To avoid order effect, the order of testing of hearing aids as well as programs P1 and P2 was randomized for each participant. The participants were instructed to repeat the stimuli heard and we scored correct responses by counting number of words correctly repeated and converted this to a percentage using the following formula:

$$
\text { SI Score }=\frac{\text { Total number of correct responses }}{\text { Total number of words presented }} \times 100
$$

\section{Ethical Considerations}

All the testing procedures carried out in the present study used the non-invasive technique, adhering to conditions for approval from the ethical committee of the institute. We explained all the test procedures to the patients and their family members before testing and received an informed consent for participating in the study from all the patients or their family members.

\section{Results}

We obtained speech identification scores were obtained from 40 ears in both unaided and four aided conditions. We calculated aided benefit by subtracting unaided SIS from aided SIS and compared all the four conditions. - Fig. 1 depicts aided benefit across the four amplification conditions compared with unaided SIS. The figure shows that the scores were higher for low-cut modified amplification strategy

Table 2 The important specifications of hearing aids used in the study

\begin{tabular}{|l|l|l|}
\hline Characteristics (Ear Simulator) & 4 channel BTE & 4 channel RIC \\
\hline Frequency Response & $100 \mathrm{~Hz}$ to $8000 \mathrm{~Hz}$ & $100 \mathrm{~Hz}$ to $8000 \mathrm{~Hz}$ \\
\hline Channels (center frequencies) & $250 \mathrm{~Hz}, 1 \mathrm{kHz}, 2.5 \mathrm{kHz}, 5 \mathrm{kHz}$ & $250 \mathrm{~Hz}, 1 \mathrm{kHz}, 2.5 \mathrm{kHz}, 5 \mathrm{kHz}$ \\
\hline Maximum gain & 68 & 65 \\
\hline MPO & 134 & 132 \\
\hline Number of Programs & 4 & 4 \\
\hline
\end{tabular}




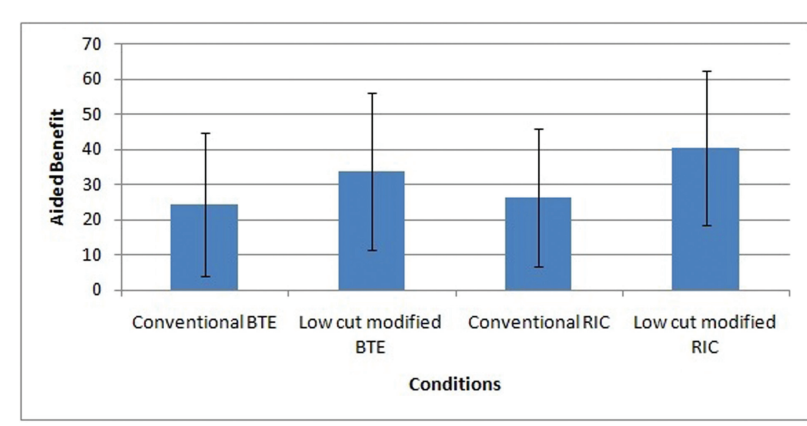

Fig. 1 Mean and standard deviation (SD) of aided benefit across different conditions.

compared with conventional amplification in both BTE and RIC. In addition, it also shows that aided benefit was higher for RIC compared with BTE with low-cut modified amplification. We also compared aided benefit obtained among good performers and poor performers with ANSD. The mean and SD of aided benefit obtained across all the four aided conditions for good and poor performers are shown in -Fig. 2 .

The unaided scores were very poor in all the participants of the study. Thus, to normalize the variance, we transformed SIS using rationalized arc sine transform ${ }^{30}$ before applying inferential statistics. Mixed ANOVA was done with conditions (4 aided conditions) as within subject factor and good/poor performers as between subject factor. The results of the study showed a significant main effect of testing conditions on $\operatorname{SIS}[\mathrm{F}(3$, $114)=54.84, \quad p<0.01]$ and significant main effect $[\mathrm{F}(1,38)=54.20, p<0.01]$ across good and poor performers. There was also a significant interaction effect $[\mathrm{F}(3,114)=44.34$, $p<0.01$ ] between conditions and across two groups. The scores obtained in all the four conditions were significantly higher $(p<0.01)$ for good performers compared with poor performers with ANSD. Further analysis using Sidak post hoc test showed that the aided benefit was significantly higher $(p<0.01)$ for low-cut modified condition compared with conventional amplification in both BTE and RIC. In addition, aided benefit with low cut modified amplification was significantly higher $(p<0.01)$ for RIC compared with BTE. However, there was a not significant difference $(p>0.05)$ in aided benefit with conventional amplification between BTE and RIC. Similar results were obtained for good performers with ANSD based on Sidak post hoc tests. However, in poor performers with ANSD, we found no

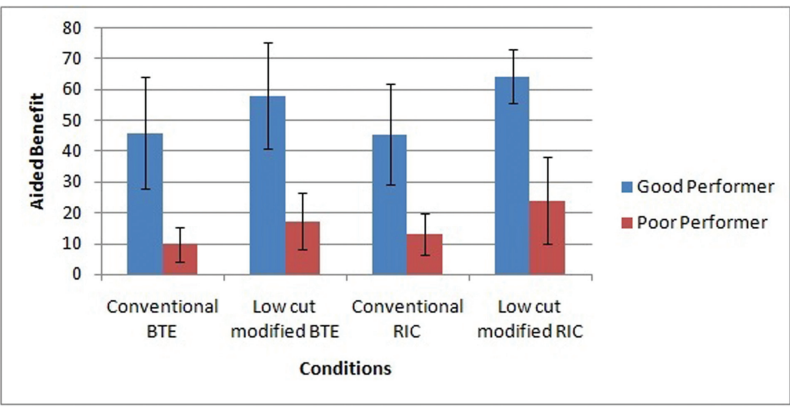

Fig. 2 Mean and standard deviation (SD) of aided benefit across different conditions in good and poor performers with ANSD.

significant difference $(p>0.05)$ in aided benefit between BTE and RIC with low-cut modified amplification. The detailed results of Sidak post hoc test are depicted in - Table 3.

\section{Discussion}

The results of the study showed that the aided performance improved with low-cut modified amplification in both BTE and RIC hearing aids (- Table 3 ). The results of the study are similar to Barman, Sinha, and Prabhu, ${ }^{12}$ who reported that low-cut modified amplification is beneficial in individuals with ANSD. They concluded that the amplified low frequency speech may mask relatively intact high frequency information. Individuals with ANSD have affected type I auditory nerve fibers, which are important to phase lock low frequency information. ${ }^{31}$ Low frequency amplification in such individuals might not help in improvement in speech perception and may even lead to the deterioration in speech identification performance. The amplified low frequency speech may mask relatively intact high frequency information. Thus, restricting access to low frequencies might prevent unwanted upward spread of masking ${ }^{32}$ of the preserved high frequency information. However, the real life benefits with low-cut modified fitting strategy need to be studied in further detail.

In the present study, we also observed improvement in aided speech perception scores with RIC compared with a BTE in the majority of individuals, as depicted in - Fig. 1. The improved clarity and naturalness of sound provided by RIC hearing aid could have led to improved aided speech perception scores in individuals with ANSD. In the RIC model, the

Table 3 The results of Sidak post hoc test and significance value for overall individuals and good/poor performers with ANSD

\begin{tabular}{|l|l|l|l|}
\hline \multicolumn{2}{|l|}{ Sidak post hoc test results } & \multicolumn{2}{l|}{ Significance Level } \\
\cline { 3 - 4 } & $\begin{array}{l}\text { Overall and Good } \\
\text { performers }\end{array}$ & Poor performers \\
\hline \multirow{2}{*}{ Conventional 4 channel BTE } & Low-cut modified amplification in 4-channel BTE & $\mathrm{p}<0.01$ & $\mathrm{p}<0.01$ \\
\cline { 2 - 4 } & Conventional 4-channel RIC & $\mathrm{P}>0.05$ & $\mathrm{P}>0.05$ \\
\cline { 2 - 4 } & Low-cut modified amplification 4-channel RIC & $\mathrm{P}<0.01$ & $\mathrm{P}<0.01$ \\
\hline \multirow{2}{*}{$\begin{array}{l}\text { Low-cut modified } \\
\text { amplification in 4 channel BTE }\end{array}$} & Conventional 4-channel RIC & $\mathrm{P}<0.01$ & $\mathrm{P}<0.01$ \\
\cline { 2 - 4 } & Low-cut modified amplification 4-channel RIC & $\mathrm{P}<0.01$ & $\mathrm{P}>0.05$ \\
\hline Conventional 4 channel RIC & Low-cut modified amplification 4-channel RIC & $\mathrm{P}<0.01$ & $\mathrm{P}<0.01$ \\
\hline
\end{tabular}


receiver is placed at the tip of the acoustic thin wire, which helps it to be fitted for a wider range of hearing loss. In the RIC hearing aid, the quality of the signal gets enhanced as it does not move through a thin wire. The separation of the receiver also leads to less feedback. The open fit used in the RIC hearing aid reduces occlusion and improves the clarity of speech. ${ }^{18,20}$ Many studies in cochlear hearing loss have demonstrated the benefit with RIC over a BTE. ${ }^{16,17}$

The improvement noticed with low-cut modified fitting with RIC was significant in good performers compared with poor performers, as shown in - Table 3 and - Fig. 2. Narne and Vanaja $^{22}$ recommended that individuals with ANSD be subdivided into good and poor performers based on speech identification scores (SIS) in quiet. The individuals were considered poor performers if the unaided speech identification scores in quiet were less than $50 \%$ and good performers if the SIS were greater than $50 \%{ }^{21}$ They reported that speech identification scores in noise were much lower for poor performers compared with good performers. This discrepancy is suspected to be because the degree of temporal distortion varies across the two groups, which determines the speech perception abilities in individuals with ANSD. Rance et $\mathrm{al}^{23}$ reported that temporal modulation transfer function results were poorer for individuals with SIS below 30\% and the scores were significantly better for children with SIS greater than $30 \%$, suggesting less temporal distortion in good performers. In the present study, the improvement was more significant with RIC, especially for good performers with ANSD. Thus, a lower degree of temporal distortion in good performers could have led to larger aided benefit. Hence, the benefit may depend on unaided speech identification scores of the client.

\section{Limitations of the Study}

The gain provided by conventional and low-cut modification was not verified using real ear probe measurements. Similar studies are essential with verification using probe microphone measurements for generalization of the results. In addition, we determined unaided scores first, followed by aided measurements. There could be practice effect which might have affected the results. In addition, it is essential to determine the real life benefit with these modifications before generalizing the results.

\section{Conclusion}

The results of the present study suggest that low-cut modification in amplification is an efficient alternative technique in contrast to conventional amplification during hearing aid fitting for individuals with ANSD. The result suggests that there could be upward spread of masking with over amplification of low frequencies in conventional amplification, which could have led to poor scores. In addition, the improvement with low-cut modified amplification was greater with RIC compared with BTE. The improved clarity and naturalness of sound with RIC could have led to better aided scores and better acceptance of the hearing aid. The result of the study also showed that greater improvement with low-cut modified amplification with RIC in good performers compared with poor performers. This suggests that the improved scores with RIC may depend on unaided SIS in quiet. Thus, low-cut modified amplification, preferably with RIC with open dome, needs to be attempted in fitting individuals with ANSD since most fail to benefit with conventional amplification strategies, especially in those with good unaided SIS in quiet.

\section{Conflict of Interest Statement}

The authors report no conflicts of interest. The authors alone are responsible for the content and writing of the paper.

\section{References}

1 Starr A, Zeng FG, Michalewski HJ, Moser T. Perspectives on Auditory Neuropathy: Disorders of Inner Hair Cell, Auditory Nerve, and Their Synapse. The Senses: A Comprehensive Reference; 2010: 397-412

2 Berlin CI, Hood LJ, Morlet T, et al. Multi-site diagnosis and management of 260 patients with auditory neuropathy/dys-synchrony (auditory neuropathy spectrum disorder). Int J Audiol 2010;49(1):30-43

3 Davis H, Hirsh SK. A slow brain stem response for low-frequency audiometry. Audiology 1979;18(6):445-461

4 Kraus N, Ozdamar O, Stein L, Reed N. Absent auditory brain stem response: peripheral hearing loss or brain stem dysfunction? Laryngoscope 1984;94(3):400-406

5 Tang TP, McPherson B, Yuen KCP, Wong LLN, Lee JSM. Auditory neuropathy/auditory dys-synchrony in school children with hearing loss: frequency of occurrence. Int J Pediatr Otorhinolaryngol 2004;68(2):175-183

6 Rance G, Beer DE, Cone-Wesson B, et al. Clinical findings for a group of infants and young children with auditory neuropathy. Ear Hear 1999;20(3):238-252

7 Kumar UA, Jayaram MM. Prevalence and audiological characteristics in individuals with auditory neuropathy/auditory dys-synchrony. Int J Audiol 2006;45(6):360-366

8 Bhat JS, Kumar K, Sinha SK. Auditory Neuropathy/Dys-synchrony In School-aged Hearing-impaired Children: A South Indian Perspective. Asia Pac J Speech Lang Hear 2007;10(3): 157-164

9 Sininger Y, Oba S. Patients with auditory neuropathy: Who are they and what can they hear? In: Sininger Y, Starr A, editors. Auditory neuropathy: A new perspective on hearing disorder. Canada: Singular Publishing Group; 2001:15-36

10 Narne VK, Prabhu P, Chandan HS, Deepthi M. Audiological profiling of 198 individuals with auditory neuropathy spectrum disorder. Hear Balance Commun 2014;12(3):112-120

11 Starr A, Picton TW, Sininger Y, Hood LJ, Berlin CI. Auditory neuropathy. Brain 1996;119(Pt 3):741-753

12 Barman A, Sinha SK, Prabhu P. Amplification strategy to enhance speech perception in individuals with auditory neuropathy spectrum disorder. Hear Balance Commun 2016;14(1):25-35

13 Zeng FG, Oba S, Garde S, Sininger Y, Starr A. Temporal and speech processing deficits in auditory neuropathy. Neuroreport 1999; 10(16):3429-3435

14 Prabhu P, Avilala V, Barman A. Speech perception abilities for spectrally modified signals in individuals with auditory dyssynchrony. Int J Audiol 2011;50(5):349-352

15 Manuel S, Barman A. Effect of spectral bandwidth and spectral integration on speech perception in individuals with normal hearing, cochlear hearing loss and auditory dyssynchrony. University of Mysore; 2012

16 Alworth LN, Plyler PN, Reber MB, Johnstone PM. The effects of receiver placement on probe microphone, performance, and 
subjective measures with open canal hearing instruments. J Am Acad Audiol 2010;21(4):249-266

17 Conrad S, Rout A. Perceived occlusion and comfort in receiver-in-the-ear hearing aids. Am J Audiol 2013;22(2): 283-290

18 Gnewikow D, Moss M. Hearing aid outcomes with open and closed canal fittings. Hear J 2006;59:66-72

19 Johnson EE. Segmenting dispensers: Factors in selecting opencanal fittings. Hear J 2006;59:58-64

20 Taylor B. Real-world satisfaction and benefit with opencanal fittings. Hear J 2006;59:74-82

21 Narne VK, Vanaja CS. Perception of envelope-enhanced speech in the presence of noise by individuals with auditory neuropathy. Ear Hear 2009;30(1):136-142

22 Narne VK, Vanaja CS. Perception of speech with envelope enhancement in individuals with auditory neuropathy and simulated loss of temporal modulation processing. Int J Audiol 2009;48(10): 700-707

23 Rance G, McKay C, Grayden D. Perceptual characterization of children with auditory neuropathy. Ear Hear 2004;25(1): $34-46$
24 Clark JG. Uses and abuses of hearing loss classification. ASHA 1981; 23(7):493-500

25 Jerger J. Clinical experience with impedance audiometry. Arch Otolaryngol 1970;92(4):311-324

26 Carhart R, Jerger JF. Preferred method for clinical determination of pure-tone thresholds. J Speech Hear Disord 1959;24:330-345

27 Kemp DT, Ryan S, Bray P. A guide to the effective use of otoacoustic emissions. Ear Hear 1990;11(2):93-105

28 Harrison WA, Norton SJ. Characteristics of transient evoked otoacoustic emissions in normal-hearing and hearing-impaired children. Ear Hear 1999;20(1):75-86

29 Manjula P, Antony J, Kumar KSS, Geetha C. Development of phonemically balanced word lists for adults in the kannada language. J Hear Sci 2015;5(1):22-30

30 Studebaker GA. A "rationalized" arcsine transform. J Speech Hear Res 1985;28(3):455-462

31 Zeng FG, Liu S. Speech perception in individuals with auditory neuropathy. J Speech Lang Hear Res 2006;49(2):367-380

32 Fabry DA, Leek MR, Walden BE, Cord M. Do adaptive frequency response (AFR) hearing aids reduce 'upward spread' of masking? J Rehabil Res Dev 1993;30(3):318-325 